\title{
Achados histológicos em 48 pacientes transplantados do fígado: biópsias do enxerto pós-reperfusão (tempo zero) e de três a 15 dias pós-transplante
}

\author{
Histological findings in 48 liver transplants: biopsies after reperfusion (time zero) and three to 15 days after \\ transplantation
}

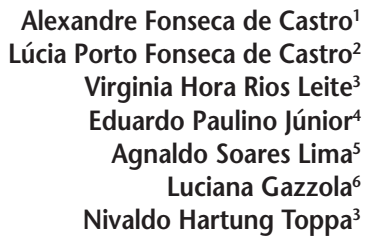

\section{unitermos resumo}

Transplante de fígado

Rejeição aguda

Endotelialite portal

Agressão ductal
Introdução: As relações entre a morfologia do enxerto transplantado e a do período pós-transplante são importantes no acompanhamento dos pacientes e no direcionamento dos tratamentos instituídos. Objetivo: Analisar os achados histológicos do enxerto hepático em biópsias realizadas pós-reperfusão (tempo zero) e naquelas realizadas de três a 15 dias pós-transplante. Materiais e métodos: Noventa e seis biópsias de 48 pacientes foram selecionadas por terem sido colhidas no tempo zero (pós-reperfusão) e no período compreendido entre o terceiro e o $15^{\circ}$ dia pós-transplante, com identificação das lesões hepatocitárias degenerativas, necrose e atividade inflamatória. As biópsias pós-transplante foram ainda graduadas quanto ao índice de atividade de rejeição (IAR), segundo o consenso de Banff. Resultados: Os achados histopatológicos mais freqüentes nas biópsias pós-reperfusão foram de degeneração hidrópica discreta (acometimento de até $50 \%$ dos hepatócitos) em $87,5 \%$ dos casos e necrose focal intralobular (lítica, apoptose) presente em $75 \%$ dos pacientes, em graus variáveis. Nas biópsias realizadas póstransplante encontrou-se degeneração hidrópica discreta também em $87,5 \%$ dos casos e rejeição aguda em 38 (79,2\%) pacientes. Nestas biópsias com rejeição aguda, chamou atenção a intensidade da agressão a ductos biliares em graus moderado (2) e acentuado (3) presentes em 42,1\% dos casos, enquanto a endotelialite portal, nestas mesmas intensidades, ocorreu em 21,05\%. Conclusão: Nossos dados evidenciaram lesões relacionadas à preservação (lesões do tipo harvesting) nas biópsias pósreperfusão. As biópsias pós-transplante revelaram índice de rejeição morfológica na maioria dos casos, como evidenciado na literatura, destacando-se aqui a intensidade da agressão a ductos biliares.

\section{abstract}

Introduction: The morphology of the donor graft and of the post-transplant period are important in the follow-up and management of liver transplanted patients. Objective: Analyse the morphological findings of the liver graft in post-reperfusional biopsies (time zero) and in biopsies taken three to 15 days after transplantation. Materials and methods: Ninety six (96) biopsies of forty eight (48) patients were selected for being performed in time zero (after reperfusion) and in the period between the $3^{\text {rd }}$ and the $15^{\text {th }}$ day after transplantation, with identification of degenerative findings, necrosis and inflamatory activity. The post-transplantation specimens were also graduated using the Rejection Activity Index (RAI), according to the Banff consensus. Results: The most common findings in time-zero biopsies were discrete hepatocyte ballooning (up to $50 \%$ of hepatocytes) in $87.5 \%$ of cases and intralobular focal necrosis (lytic, apoptosis) seen in $75 \%$ of the patients. In biopsies taken after transplantation mild hepatocyte ballooning was also seen in $87.5 \%$ of the cases. Acute rejection was seen in 38 (79.2\%) of these biopsies. Moderate (2) and intense (3) bile-duct damage was present in $42.10 \%$ of the post-transplant biopsies with acute rejection, while portal endotelialitis at the same degree of intensity was present in $21.05 \%$ of these cases.

Conclusion: Our findings reveal preservation related findings ("harvesting") in time zero biopsies. Acute rejection was seen in most cases of post-transplant specimens, according to the literature, with emphasis to the intensity of bile-duct damage. 


\section{Introdução}

O transplante hepático é hoje um procedimento difundido em todo o mundo para o tratamento de grande número de doenças do fígado. $\mathrm{O}$ aprimoramento da técnica cirúrgica, o surgimento de novos medicamentos imunossupressores e de métodos eficazes de conservação de órgãos e a melhor compreensão dos fenômenos imunológicos contribuíram de forma significativa para a redução das taxas de complicações e para o aumento da sobrevida dos pacientes transplantados (9). De fundamental importância é a análise histopatológica no transplante, útil na avaliação da viabilidade do órgão, na monitorização terapêutica e na avaliação de possíveis complicações (5, $7,8)$. A biópsia pós-reperfusão (tempo zero) permite avaliar o grau de lesões que ocorrem desde a morte encefálica do doador até o final da cirurgia do receptor (lesões de preservação), servindo como parâmetro comparativo para biópsias subseqüentes, além de possibilitar a avaliação prognóstica do enxerto. As biópsias hepáticas no período pós-transplante são úteis na identificação de complicações e no diagnóstico diferencial entre elas, sendo importantes no acompanhamento e direcionamento dos tratamentos instituídos. As relações entre os achados pós-reperfusão (tempo zero) e os do período pós-transplante são, portanto, essenciais na abordagem do paciente transplantado.

\section{Objetivo}

O objetivo do presente trabalho é analisar os achados histológicos de biópsias hepáticas que foram realizadas no tempo zero (pós-reperfusão) e os achados morfológicos do fígado do terceiro ao $15^{\circ}$ dia pós-transplante. As biópsias pós-transplante foram ainda analisadas semiquantitativamente, de acordo com o esquema de Banff (4).

\section{Materiais e métodos}

Noventa e seis biópsias hepáticas de 48 pacientes transplantados de fígado foram selecionadas no Serviço de Anatomia Patológica do Hospital das Clínicas da Universidade Federal de Minas Gerais (UFMG) e do Laboratório Analys, em Belo Horizonte, por terem sido colhidas no tempo zero (pós-reperfusão) e no período compreendido entre o terceiro e o $15^{\circ}$ dia pós-transplante. Rotineiramente, a segunda biópsia é realizada no sétimo dia pós-transplante, conforme protocolo existente no serviço. Todavia esta variação entre o terceiro e o $15^{\circ}$ dia se dá em virtude de intercorrências clínicas e/ou da disponibilidade do serviço de transplantes na execução do procedimento. As biópsias foram realizadas com agulha do tipo Hepafix 14G por um dos membros do serviço de transplantes. Os fragmentos de fígado obtidos foram fixados em solução de formol tamponado a $10 \%$ por um período mínimo de três horas e processados conforme as técnicas histológicas de rotina até a inclusão em parafina. Foram feitos vários cortes histológicos com $3 \mathrm{~mm}$ a $5 \mathrm{~mm}$ de espessura, corados por hematoxilina e eosina e tricrômico de Gomori. As lâminas foram analisadas sem o conhecimento do tempo de realização da biópsia. A análise foi feita com protocolo que considerou as lesões hepáticas gerais (degeneração hidrópica, esteatose, diferentes tipos de necrose hepatocelular e inflamação), sendo estas graduadas em discretas (acometimento de até $50 \%$ dos hepatócitos), moderadas (acometimento de $50 \%$ a $75 \%$ dos hepatócitos) ou acentuadas (acometimento de mais de 75\% dos hepatócitos). A caracterização de rejeição celular aguda (RCA) nas biópsias pós-transplante considerou a presença de no mínimo duas da tríade das lesões descritas (10) (inflamação portal mista com predomínio de células mononucleadas, contendo células blásticas, neutrófilos e freqüentemente eosinófilos; lesão de ducto biliar; endotelialite portal ou de vênula hepática terminal), sendo que estes achados foram analisados e pontuados empregando-se o índice de atividade de rejeição (IAR) do Consenso Internacional de Banff $(3,4)$. Aquelas biópsias pós-transplante em que os achados histopatológicos levaram à suspeita de outro evento predominante que não a rejeição aguda foram reanalisadas. Os critérios utilizados para selecionar estas biópsias foram a presença de agressão ductal por infiltrado predominantemente neutrofílico e sem endotelialite, ou a presença de necrose panacinar. Estas biópsias, ao contrário daquelas em que a rejeição aguda é o único evento, ou pelo menos o predominante, são especialmente difíceis de serem classificadas pelo índice de atividade de rejeição, já que outras complicações (biliares, por exemplo) podem mostrar alterações semelhantes às incluídas no esquema de classificação.

\section{Resultados}

\section{Tempo zero (pós-reperfusão)}

Os achados histológicos nas biópsias do tempo zero estão representados na Tabela 1. As alterações histológicas nestas biópsias foram de grau discreto a moderado. A degeneração hidrópica dos hepatócitos esteve presente 


\section{Achados histopatológicos nas 48 biópsias realizadas no tempo zero (pós-reperfusão) dos}

Tabela 1 pacientes submetidos a transplante hepático

\begin{tabular}{lcccccc} 
Tipos de lesão & \multicolumn{2}{c}{ Ausente } & \multicolumn{2}{c}{ Grau } \\
& \multicolumn{2}{c}{ Discreto } & \multicolumn{2}{c}{ Moderado } \\
& $n$ & $\%$ & $n$ & $\%$ & $n$ & $\%$ \\
Degeneração hidrópica & 1 & 2,1 & 42 & 87,5 & 5 & 10,4 \\
Esteatose macrovacuolar & 31 & 64,6 & 17 & 35,4 & 0 & 0 \\
Esteatose microvacuolar & 27 & 56,2 & 19 & 39,6 & 2 & 4,2 \\
Necrose focal & 12 & 25 & 33 & 68,7 & 3 & 6,3 \\
Necrose confluente focal & 47 & 97,9 & 1 & 2,1 & 0 & 0 \\
Necrose em zona 3 & 44 & 91,7 & 4 & 8,3 & 0 & 0 \\
Infiltrado inflamatório portal & 25 & 52,1 & 23 & 47,9 & 0 & 0 \\
Infiltrado inflamatório lobular & 11 & 22,9 & 35 & 72,9 & 2 & 4,2 \\
Colestase intracelular & 35 & 72,9 & 12 & 25 & 1 & 2,1 \\
Colestase intracanalicular & 47 & 97,9 & 1 & 2,1 & 0 & 0
\end{tabular}

Grau discreto = acometimento de até 50\% dos hepatócitos; grau moderado = acometimento de 50\% a 75\% dos hepatócitos; grau acentuado = acometimento de mais de $75 \%$ dos hepatócitos.

em 47 biópsias, sendo discreta em 42 casos $(87,5 \%)$ e moderada em cinco (10,4\%). A esteatose hepática, tanto macrovacuolar como microvacuolar (distribuída principalmente nas zonas 2 e 3 do ácino), foi evidenciada em grau discreto em 17 (35,4\%) e 19 (39,6\%) das biópsias, respectivamente. Em apenas dois casos a esteatose comprometia $50 \%$ a $75 \%$ dos hepatócitos, sendo de padrão microvacuolar. A necrose focal de hepatócitos foi achado freqüente $(75 \%)$, predominando a de grau discreto $(68,7 \%$ das biópsias). De um modo geral, em correspondência a estes focos de necrose, havia um infiltrado inflamatório de células polimorfonucleares também discreto $(72,9 \%$ das biópsias). A necrose de hepatócitos perivênula hepática terminal (zona 3) ocorreu em apenas quatro casos (8,3\%). A colestase intracelular foi vista em $25 \%$ dos casos, com predomínio também na zona 3. Em um caso havia colestase intracanalicular discreta pericentrolobular. Em 23 biópsias $(47,9 \%)$ havia infiltrado inflamatório portal misto de grau discreto, de células polimorfo e mononucleares.

\section{Três a 15 dias pós-transplante}

A rejeição celular aguda foi observada em 38 (79,2\%) biópsias pós-transplante. Aplicando-se o IAR de Bannf (3) a estas biópsias (Tabela 2) verificou-se que 27 (71,05\%) apresentaram rejeição discreta (IAR menor que 6); oito $(21,05 \%)$, rejeição moderada (IAR 6 e 7$)$; e três $(7,89 \%)$, rejeição acentuada (IAR 8 e 9). Entre estas biópsias em que a rejeição aguda foi o evento predominante, a endotelialite portal e a agressão ductal pelo infiltrado inflamatório
Índice de atividade de rejeição (IAR) nas biópsias hepáticas póstransplante dos pacientes em que a rejeição celular aguda estava Tabela 2 presente

\begin{tabular}{ccc}
\hline IAR & $n$ & $\%$ \\
2 & 7 & 18,42 \\
3 & 6 & 15,79 \\
4 & 4 & 10,52 \\
5 & 10 & 26,32 \\
6 & 4 & 10,52 \\
7 & 4 & 10,52 \\
8 & 3 & 7,89 \\
9 & 0 & 0 \\
\hline
\end{tabular}

tiveram intensidade variável, como pode ser observado na Tabela 3. A endotelialite portal foi identificada (em graus variáveis) em $32(84,2 \%)$ dos 38 pacientes, sendo de grau moderado ou acentuado em oito (21,05\%). A agressão ductal pelo infiltrado inflamatório ocorreu em 34 $(89,47 \%)$ destes pacientes, sendo de grau moderado ou acentuado em 16 (42,10\%).

Em dez (20,8\%) das 48 biópsias, a rejeição aguda não esteve presente ou não foi o evento predominante (critérios de seleção já discutidos na seção Materiais e Métodos): cinco $(10,42 \%)$ mostraram apenas alterações inespecíficas compatíveis com lesões do tipo harvesting, com degeneração hidrópica, esteatose macrovacuolar e 


\begin{tabular}{lcccc|} 
& \multicolumn{2}{c|}{$\begin{array}{c}\text { Endotelialite portal e agressão } \\
\text { ductal encontradas nas biópsias } \\
\text { Tabela }\end{array}$} \\
hepáticas com rejeição celular aguda
\end{tabular}

esteatose microvacuolar em graus semelhantes aos observados no tempo zero; quatro $(8,33 \%)$ evidenciaram necrose panacinar, compatível com alteração de caráter isquêmico; um apresentou inflamação portal predominantemente neutrofílica com agressão ductal pelo infiltrado e colestase intracelular e intracanalicular, sem nenhum grau de endotelialite, quadro compatível com complicação biliar sem rejeição aguda. Os achados histológicos nas 48 biópsias pós-transplante, sem discriminação por diagnóstico, estão agrupados na Tabela 4.

\section{Discussão}

A biópsia hepática é um exame importante na propedêutica de monitoramento do fígado transplantado. Nos grandes centros de transplante a biópsia é feita de acordo com um protocolo preestabelecido (12). No Hospital das Clínicas da UFMG realiza-se a biópsia hepática logo após a reperfusão do órgão (tempo zero) e no sétimo dia póstransplante. Todavia ela pode ser feita em qualquer momento em que o paciente apresente manifestações clínicas e/ou alterações laboratoriais da função hepática. A avaliação histológica da biópsia no tempo zero permite não somente identificar alterações preexistentes no fígado do doador, como também as decorrentes da preservação do órgão, servindo ainda como parâmetro para análise das biópsias subseqüentes. No presente trabalho pode-se constatar que, no tempo zero, as 48 biópsias hepáticas apresentaram como lesões mais freqüentes: degeneração hidrópica de hepatócitos, esteatoses macro e microvacuolar, agregados intralobulares de neutrófilos, necrose hepatocelular focal e colestase intracitoplasmática. Estas Tabela 4 plante dos pacientes submetidos a transplante hepático

\begin{tabular}{|c|c|c|c|c|c|c|c|c|}
\hline \multirow[t]{3}{*}{ Tipos de lesão } & \multicolumn{8}{|c|}{ Grau } \\
\hline & \multicolumn{2}{|c|}{ Ausente } & \multicolumn{2}{|c|}{ Discreto } & \multicolumn{2}{|c|}{ Moderado } & \multicolumn{2}{|c|}{ Acentuado } \\
\hline & $n$ & $\%$ & $n$ & $\%$ & $n$ & $\%$ & $n$ & $\%$ \\
\hline Degeneração hidrópica & 4 & 8,3 & 42 & 87,5 & 2 & 4,2 & 0 & 0 \\
\hline Esteatose macrovacuolar & 32 & 66,7 & 15 & 31,2 & 1 & 2,1 & 0 & 0 \\
\hline Esteatose microvacuolar & 32 & 66,7 & 13 & 27,1 & 3 & 6,2 & 0 & 0 \\
\hline Necrose focal & 3 & 6,3 & 40 & 83,3 & 5 & 10,4 & 0 & 0 \\
\hline Necrose em saca-bocados & 36 & 75,0 & 10 & 20,8 & 2 & 4,2 & 0 & 0 \\
\hline Necrose confluente focal & 41 & 85,4 & 7 & 14,6 & 0 & 0 & 0 & 0 \\
\hline Necrose em zona 3 & 39 & 81,2 & 8 & 16,7 & 1 & 2,1 & 0 & 0 \\
\hline Necrose em zona 3 com pontes ocasionais & 45 & 93,7 & 2 & 4,2 & 1 & 2,1 & 0 & 0 \\
\hline Necrose em zona 3 com múltiplas pontes & 47 & 97,9 & 1 & 2,1 & 0 & 0 & 0 & 0 \\
\hline Necrose panacinar ou multiacinar & 44 & 91,7 & 0 & 0 & 4 & 8,3 & 0 & 0 \\
\hline Infiltrado inflamatório portal & 4 & 8,3 & 19 & 39,6 & 25 & 52,1 & 0 & 0 \\
\hline Infiltrado inflamatório lobular & 4 & 8,3 & 38 & 79,2 & 6 & 12,5 & 0 & 0 \\
\hline Colestase intracelular & 4 & 8,3 & 34 & 70,8 & 10 & 20,8 & 0 & 0 \\
\hline Colestase intracanalicular & 10 & 20,8 & 23 & 47,9 & 15 & 31,3 & 0 & 0 \\
\hline Endotelialite portal & 14 & 29,2 & 26 & 54,2 & 7 & 14,6 & 1 & 2,1 \\
\hline Endotelialite de vênula hepática terminal & 30 & 62,5 & 13 & 27,1 & 5 & 10,4 & 0 & 0 \\
\hline Agressão ductal pelo infiltrado inflamatório & 10 & 20,8 & 20 & 41,7 & 14 & 29,2 & 4 & 8,3 \\
\hline Degeneração de células ductais & 14 & 29,2 & 22 & 45,8 & 11 & 22,9 & 1 & 2,1 \\
\hline Necrose do epitélio ductal & 36 & 75 & 11 & 22,9 & 1 & 2,1 & 0 & 0 \\
\hline
\end{tabular}

Grau discreto = acometimento de até 50\% dos hepatócitos; grau moderado = acometimento de 50\% a 75\% dos hepatócitos; grau acentuado = acometimento de mais de $75 \%$ dos hepatócitos. 
alterações foram semelhantes à descritas na literatura $(6-8,10)$ e, em sua maioria, discretas e localizadas na zona 3 do ácino hepático. É possível que estas alterações se desenvolvam desde a fase agônica do doador até o momento de implantação e reperfusão do órgão (8). A degeneração hidrópica, a esteatose microvesicular e a colestase são denominadas lesões de preservação (lesões do tipo harvesting). Usualmente são decorrentes da isquemia a que o fígado é submetido, porém outros fatores como as drogas utilizadas e toxinas também podem causálas $(8,10,11)$. Os agregados intralobulares e portais de neutrófilos observados em $77,08 \%$ das biópsias são atribuídos à manipulação do órgão durante o procedimento, sendo esta alteração denominada "hepatite cirúrgica" (8). As alterações descritas no tempo zero são reversíveis e em geral desaparecem uma semana após o transplante. Todavia estas lesões, quando mais intensas, podem levar à disfunção grave ou ao não-funcionamento do fígado enxertado (5). A esteatose macrovacuolar foi discreta e esteve presente em 17 casos (35,42\%). É possível que este achado já estivesse presente no fígado do doador antes da retirada do órgão, uma vez que sua patogênese envolve múltiplos fatores. A presença de esteatose macrovacuolar no fígado do doador deve ser quantificada, pois os grandes vacúolos de gordura interferem na perfusão do órgão (6). Admite-se também que os lípides intracelulares podem ativar fosfolipases com formação de radicais livres, exacerbando qualquer lesão de reperfusão (6). Um fígado com esteatose macrovacuolar comprometendo $60 \%$ a $70 \%$ dos hepatócitos não deve ser utilizado, uma vez que é grande o risco de não-funcionamento do enxerto (8). As biópsias realizadas entre o terceiro e o $15^{\circ}$ dia póstransplante apresentaram rejeição celular aguda em 38 casos $(79,2 \%)$, a maioria de grau discreto $(71,05 \%)$. A RCA é um evento muito freqüente no transplante hepático, sendo diagnosticada em cerca de $70 \%$ dos casos no primeiro mês pós-transplante (10). O diagnóstico histológico de RCA é baseado na seguinte tríade: infiltrado inflamatório portal misto, com predomínio de linfócitos ativados (blastos), neutrófilos e eosinófilos; endotelialite das veias portais e/ou hepáticas terminais; lesões do epitélio ductal (Figura 1). Para o diagnóstico de RCA é necessária obrigatoriamente a presença de pelo menos dois destes achados. Uma vez caracterizado, aplica-se o sistema de Banff (4) para a sua graduação. A evidência de RCA é muito importante, pois ela pode ser controlada com o uso de imunossupressores. Entre os achados histológicos que compõem a tríade diagnóstica de RCA, o infiltrado inflamatório portal é o hallmark, e esteve presente em 44 casos $(91,7 \%)$. A endotelialite em graus variáveis foi observada em 32 casos (84,21\%), e a lesão ductal, em 34 $(89,47 \%)$. Estas alterações em graus moderado a acentuado foram observadas nos ductos biliares em 16 casos $(42,1 \%)$ e nos vasos portais, em oito $(21,05 \%)$. A maior intensidade destas lesões ductais pode ser atribuída à concomitância de outros eventos, como a isquemia arterial e/ou complicações biliares $(7,10)$. O seguimento desses pacientes, acompanhado de tratamento imunossupressor e de exames complementares, poderá dizer se a lesão ductal é de fato devida apenas à rejeição. Apesar de encontrarmos uma alta freqüência de lesão ductal, a endotelialite é o achado mais específico da RCA (10). Apesar disso, deve-se ficar atento para não superestimála, uma vez que, para o diagnóstico de endotelialite, há a necessidade de linfócito ocupar o espaço subendotelial, de modo que estas células e o endotélio façam protrusão para a luz vascular (Figuras 2 e 3). Em suma, a incidência de eventos concomitantes à rejeição que exacerbam a lesão ductal, a superestimação da endotelialite e outras dife-

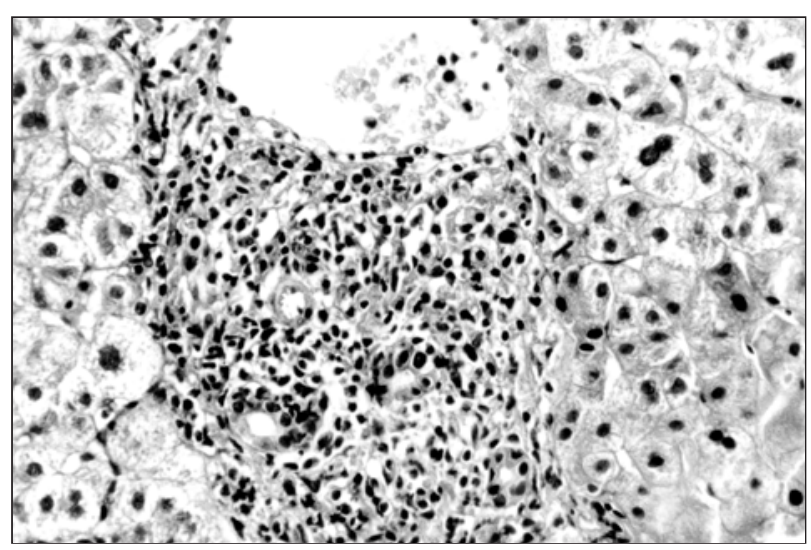

Figura 1 - Infiltrado inflamatório portal misto, endotelialite e agressão ductal: tríade da rejeição celular aguda (400x)

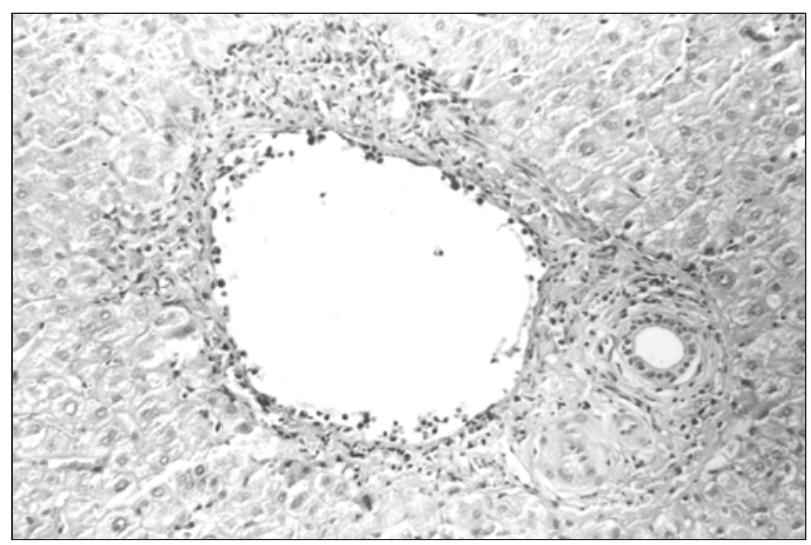

Figura 2 - Endotelialite portal: notar linfócitos ocupando o espaço subendotelial e fazendo saliência para a luz do vaso (200x) 
renças em critérios utilizados na graduação das lesões podem contribuir para a variação na intensidade dos achados de RCA encontrados na literatura $(2,10)$. A presença de outras alterações encontradas nas biópsias realizadas entre o terceiro e o $15^{\circ}$ dia pós-transplante como esteatose, degeneração hidrópica, necrose hepatocelular focal e colestase foi discreta e, possivelmente, secundária à preservação do órgão.

\section{Conclusão}

A biópsia hepática no tempo zero (pós-reperfusão) mostrou-se importante na detecção de lesões preexistentes no fígado do doador e de lesões relacionadas à preservação (lesões do tipo harvesting). No presente trabalho, estas lesões foram discretas e semelhantes às descritas na literatura. As biópsias hepáticas do terceiro ao $15^{\circ}$ dia pós-

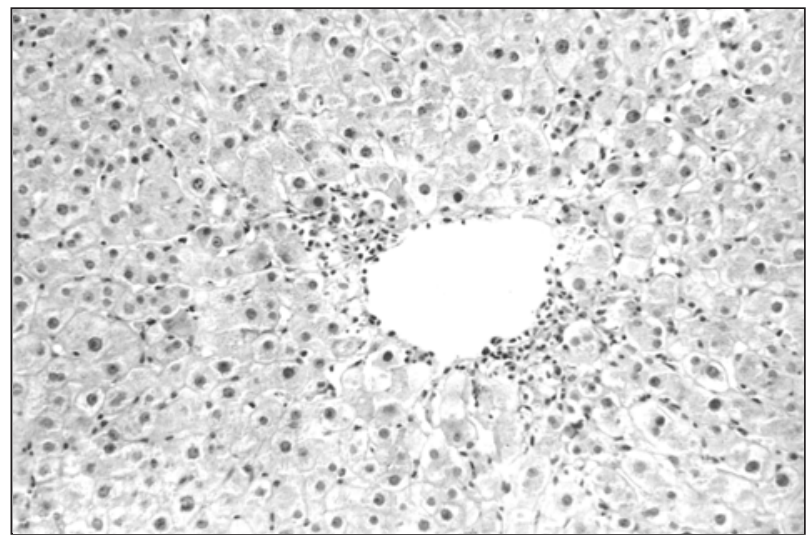

Figura 3 - Endotelialite em vênula hepática terminal (200x)

transplante mostraram, em sua maioria, lesões de rejeição celular aguda. A endotelialite e a lesão ductal estiveram presentes na maior parte das biópsias em proporções semelhantes, porém a lesão ductal foi mais intensa.

\section{Referências}

I. Demetris, A.J. Acute liver allograft rejection and its differential diagnosis pathology. In: Blommer J. R., Goodman Z. D., Ischak K.G. (eds.) Clinical and pathologycal correlations in liver disease: approaching the next millennium. The Armed Forces Institute of Pathology, The American Registry of Pathology, The American Association for the Study of Liver Diseases, 1998 , p. |08-2|.

2. Demetris, A.J. et al. Intraobserver and interobserver variation in the histopathological assessment of liver allograft rejection. Hepatology, I 4(5): 751-5, 199 |

3. Demetris, A.J. et al., for the International Working Party. Terminology for hepatic allograft rejection. Hepatology, 22 (2): 648-54, 1995

4. Demetris, A.J. et al. Banff schema for grading liver allograft rejection: An international consensus document, 25(3): 658-63, 1997.

5. Gayotto, L.C.C. \& Mello E. S. Patologia hepática. Hand out, Curso Curto (CCI4), XXII Congresso Brasileiro de Patologia, 1999.

6. Lemasters, J..; Bunzendahl, H. \& Thurman, R.G. Preservation of the liver. In: Maddrey, W.C.; Schiff, E.R. \& Sonell, M.F. (eds.)
Transplantation of the Liver. 3. ed. Lippincott: Williams \& Wilkins, 200 I, p. 250-3.

7. Ludwig, J.\& Lefkowitch, J.H. Histopathology of the liver following transplantation. In: Maddrey, W.C.; Schiff, E.R. \& Sonell, M.F. (eds.) Transplantation of the Liver. 3. ed. Lippincott: Williams \&Wilkins, 200 I, p. 229-50.

8. Portmann, B. \& Koukoulis, G. Pathology of the liver allograft. Curr. Top. Pathol., 92: 61-105, 1999.

9. Schafer, D.F. Looking back, looking forward. In: Maddrey, W.C.; Shciff, E. R. \& Sonell, M. F. (eds.) Transplantation of the liver. $3^{\text {rd }}$ ed. Lippincott:Williams \& Wilkins, 200 I, p. I-3.

10. Snover, D.C. Problems in the interpretation of liver biopsies after liver transplantation. Am. J. Clin Pathol., I 3(1): 31-8, 1989.

I I. Snover, D.C. et al. Acute rejection in human liver grafts: A comparative study of cases maintained on azathioprine and prednisone versus ciclosporine $A$ and low-dose steroids. Hum. Pathol., 19(9): 1036-42, 1988.

12. Wight, D.G.D. Aspects of liver transplant pathology with emphasis on rejection and its mechanisms. J. Clin. Pathol., 47(4): 296-9, 1994.
Endereço para correspondência

Lúcia Porto Fonseca de Castro Rua Cláudio Manoel 99/101

Bairro Funcionários

CEP 30140-100 - Belo Horizonte-MG

e-mail: lupato@@terra.com.br lecoar@hotmail.com 